\title{
A Case report of Recurrent Vomiting: Extending the Spectrum of Neuronal Intranuclear Inclusion Disease
}

\section{Xiaoyun Liu}

Shandong Provincial Hospital affiliated to Shandong University https://orcid.org/0000-0003-08546190

Xiaohui Liu

Shandong Provincial Hospital affiliated to Shandong University

Yifeng Du

Shandong Provincial Hospital affiliated to Shandong University

\section{Chunxia Li}

Shandong Provincial Hospital affiliated to Shandong University

Cuicui liu

Shandong Provincial Hospital affiliated to Shandong Provincial Hospital

Lin Lu ( $\nabla$ lulin558@163.com )

https://orcid.org/0000-0002-2840-4635

\section{Case report}

Keywords: Neuronal intranuclear inclusion disease; recurrent vomiting; skin biopsy; electron microscope; diffusion-weighted imaging

Posted Date: April 4th, 2019

DOI: https://doi.org/10.21203/rs.2.1814/v1

License: (c) (1) This work is licensed under a Creative Commons Attribution 4.0 International License. Read Full License 


\section{Abstract}

Background Neuronal intranuclear inclusion disease (NIID) is a recently defined disease entity of progressive neurodegenerative disease with characterizations of eosinophilic hyaline intranuclear inclusions in neuronal and somatic cells. The sporadic adult-onset NIID cases were previous described as 'dementia dominant group'. Here we present a NIID case manifested prominently with recurrent vomiting. Case presentation A 60-year-old women present with paroxysmal vomiting, hypertention and decreased level of consciousness for 3 years. She was diagnosed with NIID based on history, clinical features, brain magnetic resonance imaging(MRI), skin biopsy. Conclusion Autonomic symptoms may manifest as the initial and predominant presentation of NIID. This case presentation may extend the spectrum of NIID and may give new insights in exploring the pathogenic mechanisms of NIID.

\section{Introduction}

Neuronal intranuclear inclusion disease (NIID) is a rare progressive neurodegenerative disorder characterized by eosinophilic hyaline intranuclear inclusions presented in the central and peripheral nervous systems, and in the visceral organs[1]. Recently, it has been found that skin biopsy is an effective and less invasive ante-mortem diagnostic tool for NIID and shows similar pathological changes to postmortem dissection[2]. The clinical manifestations differs among patients and can be classified into 3 categories according to the age of onset: infantile, juvenile, and adult forms[1]. Here we report a sporadic adult-onset NIID case mainly manifested as recurrent vomiting, hypertention and decreased level of consciousness.

\section{Case Presentation}

A 60-year-old Chinese woman, who was used to be a farmer, was admitted to our medical center due to recurrent vomiting and decreased level of consciousness for the past three years and the symptoms recurred during the recent 12 days. 3 years before administration, the patient developed nausea and vomiting coupled with decreased level of consciousness, elevated blood pressure, urinary incontinence, somnolence and mutism. During the episode of symptoms, blood pressure elevated to $150 \sim 170 / 80 \sim 100 \mathrm{mmHg}$ from baseline of $125 / 75 \mathrm{mmHg}$. There were not any prodromal symptoms of fever, dizziness or headache before disease episode. Symptoms appeared periodically for every half a month or 2 months and lasted for about 7 10 days and could be relieved after usual supportive treatment. She became slow in response speed from 2 years ago. 12 days before administration, the patient had similar symptoms with longer time. She had a past medical history of diabetes mellitus, cataract and family history of fundus macular hole.

The patient has a poor health condition with a body weight index of $17.6 \mathrm{~kg} / \mathrm{m}^{2}$. Physical examination revealed blood pressure of $130 / 84 \mathrm{mmHg}$ and body temperature of $36.1^{\circ} \mathrm{C}$. The neurological examination revealed that she was dysphoric and did not respond to external stimulation. Her right pupil was irregular after cataract surgery and left pupil was normal. Fine nystagmus was noticed in her right 
eye. Deep reflexes, including biceps reflex, triceps reflex, patellar reflex and Achilles reflex disappeared. Active movement of limbs were visible and muscular tone was decreased. Both Babinski sign and Chaddock sign were positive bilaterally.

Cranial computed tomography (CT) scan performed 3 years ago immediately after her first clinical episode showed diffuse low density in the bilateral paraventricular white matter. Further examinations were proposed to clarify the diagnosis after her administration. Laboratory examinations revealed elevated blood glucose of $7.17 \mathrm{mmol} / \mathrm{L}$, decreased albumin of $35.8 \mathrm{~g} / \mathrm{L}$ and a $\mathrm{HbA} 1 \mathrm{c}$ concentration of $7.3 \%$. Lumbar puncture was performed and the opening pressure was $140 \mathrm{cmH} 20$. Cerebrospinal fluid examination revealed no pleocytosis or elevated protein level and normal glucose level. Complete blood count, liver and renal functions, antinuclear antibody spectrum, ANCA series were normal. Viral markers, syphilis test, catecholamines, hematuria organic acid were negative. Enhanced adrenal CT scan did not find hyperplasia or adenoma. Brain magnetic resonance imaging (MRI) revealed mild cerebral atrophy and moderate cerebellar atrophy, and showed high intensity in the cerebral white matter on T2-weighted imaging, fluid-attenuated inversion recovery (FLAIR) and in corticomedullary junction on diffusionweighted imaging. The video electroencephalogram (EEG) showed asymmetrical posterior rhythm with lower amplitude on the left side. Nerve conduction studies showed a multiple and symmetrical reduction in both motor and sensory nerve conduction velocity. Screening of thoracic CT scan, abdominal and gynecological ultrasound did not find sign of tumor. Skin biopsy performed in left leg showed chronic inflammatory cells infiltration in hematoxylin and eosin staining and intranuclear inclusions in adipocytes, sweat gland cells and fibroblasts in immunohistochemical staining and under electron microscope.

Three days after nutrition support therapy, the patient regained consciousness and was able to communicate in language, while the memory and understanding was impaired. Her mini mental state examination (MMSE) score and frontal assessment battery (FAB) score were 12/30 (illiterate) and 4 (14.5/18), respectively. Mild weakness was present in both upper and lower limbs. Algesthesia, pallesthesia, topognosis were abated in the right side. Coordinate movements were steady and accurate. Orthostatic hypotension was observed in Schellong test, with a decrement of systolic blood pressure by $22 \mathrm{mmH}_{2} \mathrm{O}$. The fragile $\mathrm{X}$ mental retardation1 (FMR1) permutation repeats of CGG was in normal range. With all the data from clinical examination listed above, we diagnosed her with adult-onset neuronal intranuclear inclusion disease(NIID).

\section{Discussion}

Autonomic symptoms are the initial and main clinical characters of this patient, including paroxysmal nausea, vomiting and hypertention following recurrent brief loss of conscious, and orthostatic hypotension, other symptoms such as cognitive impairment, peripheral neuropathy, mild muscle weakness, sensory disturbance are also presented. The typical linear high intensity signals in the 
corticomedullary junction on DWI images triggers the skin biopsy, which reveals ubiquitin and P62 positive intranuclear inclusions also visible under an electron microscope. We diagnosed this patient as a sporadic adult-onset NIID by the combination of clinical symptoms, characteristic DWI signals, intranuclear inclusions and the negative of FMRI gene premutation.

NIID is a rare neurodegenerative disease with highly variable clinical manifestations. Adult-onset NIID includes sporadic and familial NIID, and recently the clinicopathological features were described[3]. Previously, the highly variable clinical manifestations of NIID renders the ante-mortem diagnosis to be difficult, and medical professionals relay on the typical corticomedullary high intensity on DWI image as a strong indicator of the ante-mortem diagnosis of NIID [3, 4]. Recently, it has been found that skin biopsy is an effective and less invasive ante-mortem diagnostic tool for NIID and shows similar pathological changes to post-mortem dissection[2]. The onset age of sporadic cases is between 51 and 76 years old, and the disease duration ranges from 1 to 19 years. Most sporadic NIID cases (94.7\%) presented dementia as the initial and main clinical manifestation and were recognized as dementia dominant group. Miosis and bladder dysfunction manifesting as urinary incontinence were the most common autonomic impairments. Vomiting was also present in some $(15.8 \%)$ of these patients, but rarely considered as the initial and presenting symptom. There were also case reports of sporadic and familial adult-onset NIID patients presenting bladder dysfunction, fecal incontinence or orthostatic hypotension as the initial symptoms $[5,6]$. With that, we propose there is another subtype of autonomic dominant group in addition to the dementia dominant group in those sporadic adult-onset NIID patients.

Despite the diagnosis efforts $邓$ the pathogenesis of NIID remains unclear. Nuclear inclusions (NIs) are not restricted to the central nervous system, but are also distributed in peripheral nerves systems and visceral organs $[7,8]$. NIs in the skin biopsy specimens show similar imunopositivity for ubiquitin and p62 to the central nervous system $[4,9]$. Nls are formed when there is excessive accumulation of proteins in the nucleus and the abnormal alteration of nuclear bodies might be related to the pathogenesis of NIID $[10,11]$. Excessive protein accumulation in Nls might impair the ubiquitin-dependent degradation process and consequently result in dysfunction of neurons or somatic cells, however, further pathological and molecular studies will be necessary to fully understand the pathogenesis of NIID.

The prevalence rate of adult-onset NIID may be higher than previously thought, and that NIID may be underdiagnosed[3]. We present a sporadic adult-onset NIID case with autonomic symptom as initial and main clinical characters and propose that there may be another subtype of autonomic dominant group in addition to the dementia dominant group in those sporadic adult-onset NIID patients. DWI images and skin biopsy are critical in the correct diagnosis of NIID and should be considered as an essential step during diagnosis.

\section{Abbreviations}

NIID: Neuronal intranuclear inclusion disease; MRI: Magnetic resonance imaging; CT: Computed tomography; FLAIR: Fluid-attenuated inversion recovery $\mathbb{D}$ DIID iffusion-weighted imaging $\triangle A N C A$ : Anti- 


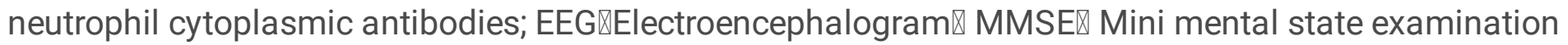

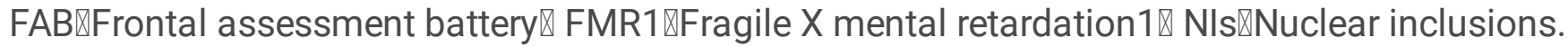

\section{Declarations}

\section{Ethics approval and consent to participate}

This study was approved by the Institutional Review Board of Shandong Provincial Hospital Affiliated to Shandong University. Informed consent was obtained from the patient.

\section{Consent to publish}

Written informed consent was obtained from the patient for publication of this case report and any accompanying images. A copy of the written consent is available for review by the editor of this journal.

\section{Availability of data and materials}

All data and material supporting our findings are contained within the manuscript and additional supporting files.

\section{Competing interests}

The authors declare no conflicts of interest with respect to the research, authorship, and/or publication of this article.

\section{Funding}

No funding support.

\section{Authors' contributions}

$X Y L$ and LL participated the design of this case report. XYL, XHL,YFD,CCL,CXL and LL collected and analyzed the raw clinical data. XYL wrote the manuscript. All authors have read and approved the final manuscript.

\section{Acknowledgements}

The authors are grateful to the patient for her participation in our study. This case report was completed by the efforts of all authors.

\section{References}

1. J, T.-F., Neuronal intranuclear hyaline inclusion disease. Neuropathology Official Journal the Japanese Society of Neuropathology, 2003. 23. 
2. Sone, J., et al., Skin biopsy is useful for the antemortem diagnosis of neuronal intranuclear inclusion disease. Neurology, 2011. 76(16): p. 1372-6.

3. Sone, J., et al., Clinicopathological features of adult-onset neuronal intranuclear inclusion disease. Brain, 2016. 139(Pt 12): p. 3170-3186.

4. Sone, J., et al., Neuronal intranuclear inclusion disease cases with leukoencephalopathy diagnosed via skin biopsy. Journal of Neurology Neurosurgery \& Psychiatry, 2014. 85(3): p. 354.

5. Zannolli, R., et al., Hereditary neuronal intranuclear inclusion disease with autonomic failure and cerebellar degeneration. Archives of Neurology, 2002. 59(8): p. 1319-26.

6. Nakamura, M., et al., Two cases of sporadic adult-onset neuronal intranuclear inclusion disease preceded by urinary disturbance for many years. J Neurol Sci, 2018. 392: p. 89-93.

7. Lindenberg, R., et al., A light and electron microscopy study of an unusual widespread nuclear inclusion body disease. Acta Neuropathologica, 1968. 10(1): p. 54-73.

8. Tateishi, J., et al., Intranuclear inclusions in muscle, nervous tissue, and adrenal gland. Acta Neuropathologica, 1984. 63(1): p. 24-32.

9. Mori, F., et al., Incipient intranuclear inclusion body disease in a 78-year-old woman. Neuropathology, 2011. 31(2): p. 188-193.

10. Takahashi-Fujigasaki, J., et al., SUMOylation substrates in neuronal intranuclear inclusion disease. Neuropathology \& Applied Neurobiology, 2010. 32(1): p. 92-100.

11. Nakano, Y., et al., PML Nuclear Bodies Are Altered in Adult-Onset Neuronal Intranuclear Hyaline Inclusion Disease. J Neuropathol Exp Neurol, 2017. 76(7): p. 585-594.

\section{Figures}

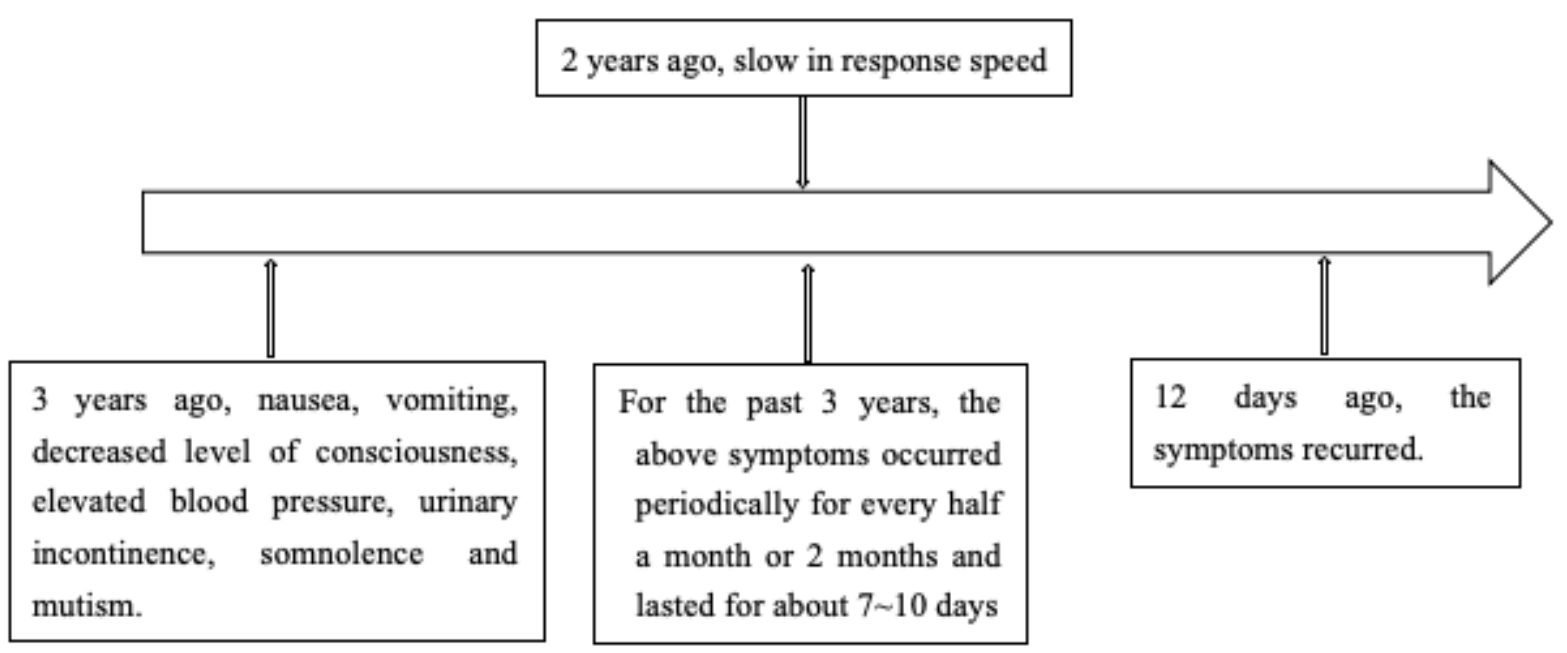

\section{Figure 1}

Timeline of the patient. 

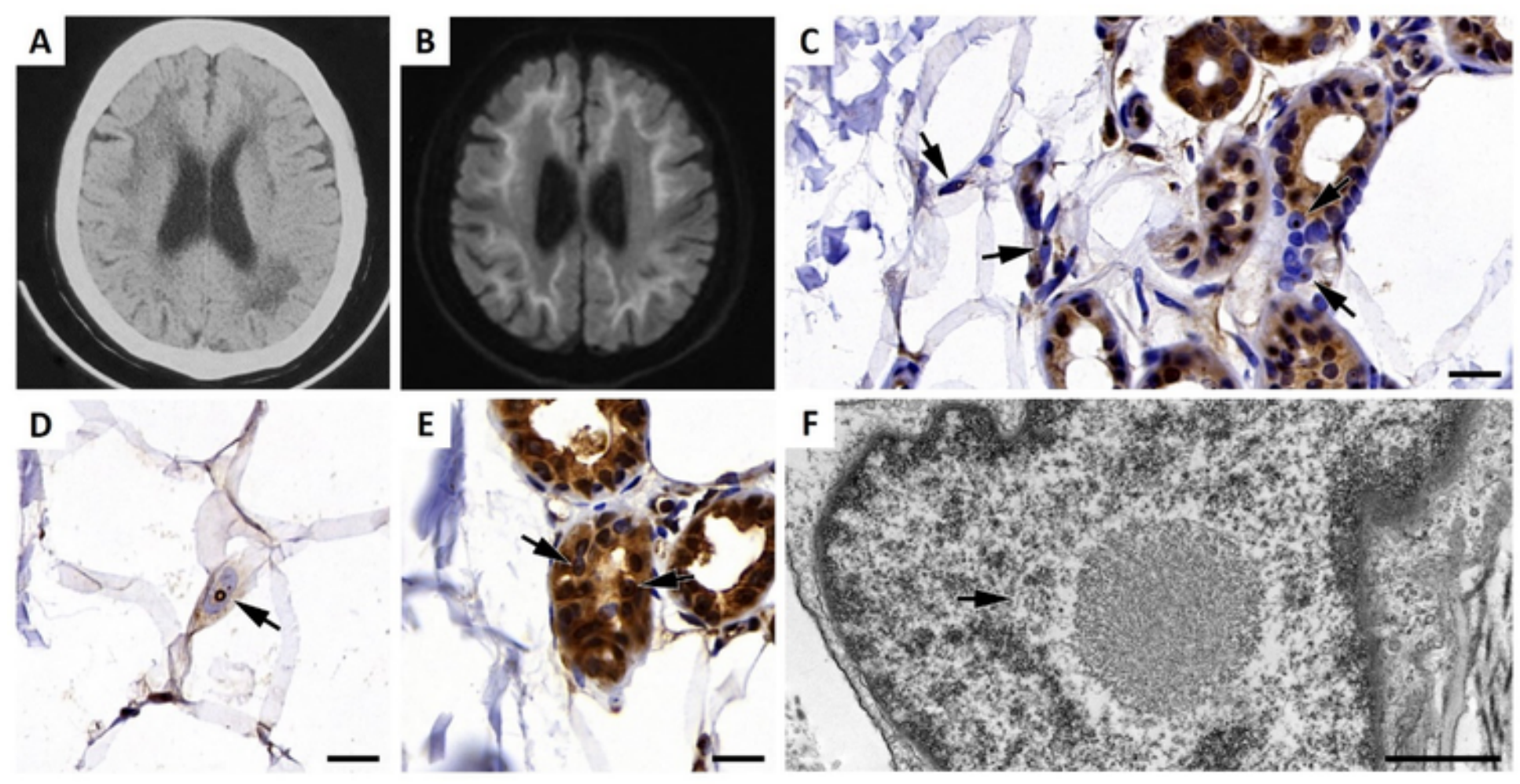

Figure 2

A Cranial CT at disease onset showing diffuse low density in the bilateral paraventricular white matter; B DWI imaging 3 years after disease onset showing a linear high-intensity signals alone the corticomedullary junction; $\mathrm{C}$ Immunohistochemical staining of P62, the left 2 arrows indicate intranuclear inclusions in fibroblasts, the right 2 arrows indicate intranuclear inclusions in sweat gland cells; bar=20um; D Immunohistochemical staining of P62, the arrow indicates intranuclear inclusion in adipocyte; bar=20um; E Immunohistochemical staining of ubiquitin, the arrows indicate intranuclear inclusions in sweat gland cells; bar=20um; F Electron microscopic image of fibroblast showing intranuclear inclusions; bar=1um.

\section{Supplementary Files}

This is a list of supplementary files associated with this preprint. Click to download.

- supplement1.docx 